\title{
BMJ The relationship between osteoarthritis Open and cardiovascular disease in a population health survey: a cross-sectional study
}

\author{
M Mushfiqur Rahman, ${ }^{1,2}$ Jacek A Kopec, ${ }^{1,2}$ Jolanda Cibere, ${ }^{2,3}$ \\ Charlie H Goldsmith, ${ }^{2,4}$ Aslam H Anis ${ }^{1,5}$
}

To cite: Rahman MM, Kopec JA, Cibere J, et al. The relationship between osteoarthritis and cardiovascular disease in a population health survey: a cross-sectional study. BMJ Open 2013;3:e002624. doi:10.1136/bmjopen-2013002624

- Prepublication history for this paper are available online. To view these files please visit the journal online (http://dx.doi.org/10.1136/ bmjopen-2013-002624).

Received 23 January 2013 Revised 14 April 2013 Accepted 16 April 2013

This final article is available for use under the terms of the Creative Commons Attribution Non-Commercial 2.0 Licence; see http://bmjopen.bmj.com

For numbered affiliations see end of article.

Correspondence to Mr M Mushfiqur Rahman; mrahman@arthritisresearch.ca

\section{ABSTRACT}

Objectives: Our objective was to determine the relationship between osteoarthritis $(\mathrm{OA})$ and heart diseases (myocardial infarction (MI), angina, congestive heart failure (CHF)) and stroke using population-based survey data.

Design: Cross-sectional study.

Setting: Canadian Community Health Survey (CCHS). Participants: Adult participants in the CCHS cycles 1.1, 2.1 and 3.1 were included. CCHS provides nationally representative data on health determinants, health status and health system utilisation. We have identified 40817 self-reported $O A$ subjects and selected 1:1 matched non-OA respondents by age, sex and CCHS cycles.

Main outcome measures: Self-reported heart disease was the primary outcome and $\mathrm{MI}$, angina, $\mathrm{CHF}$ and stroke were considered as secondary outcomes.

Multivariable logistic regression models were used to estimate the ORs after adjusting for sociodemographic status, obesity, physical activity, smoking status, fruit and vegetable consumption, medication use, diabetes, hypertension and chronic obstructive pulmonary disease.

Results: The mean age of $\mathrm{OA}$ cases was 66 years and $71.6 \%$ were women. OA exhibited increased odds of prevalent heart disease, and adjusted overall OR $(95 \% \mathrm{Cl})$ was 1.45 (1.36 to 1.54), 1.35 (1.21 to 1.50) among men and 1.51 (1.39 to 1.64) among women with $\mathrm{OA}$. OA showed increased ORs for angina and CHF in both men and women, and for $\mathrm{MI}$ in women. ORs $(95 \% \mathrm{Cl})$ for men and women, respectively, were 1.08 (0.91 to 1.28) and 1.49 (1.28 to 1.75) for MI, 1.76 (1.43 to 2.17) and 1.84 (1.59 to 2.14) for angina, 1.50 (1.13 to 1.97 ) and 1.81 (1.49 to 2.21) for CHF, and 1.08 (0.83 to 1.40 ) and 1.13 ( 0.93 to 1.37 ) for stroke.

Conclusions: Prevalent $0 \mathrm{~A}$ was associated with selfreported heart disease, particularly angina, and $\mathrm{CHF}$ in both men and women, after controlling for established risk factors for these conditions. This study provides a rationale for further investigation of the association between $O A$ and heart disease in longitudinal studies for investigating possible biological and behavioural mechanisms.

\section{ARTICLE SUMMARY}

Article focus

- The purpose of this study was to determine the association between osteoarthritis $(\mathrm{OA})$ and cardiovascular disease (CVD) using data from a large population survey in Canada.

- We analysed the association between $O A$ and CVD, myocardial infarction (MI), angina, congestive heart failure (CHF) and stroke.

- All analyses were carried out for the entire population and separately for men and women.

Key messages

- OA was significantly associated with any heart disease, angina and CHF in both men and women after controlling for potential confounders.

- We observed that OA was significantly associated with $\mathrm{Ml}$ among women only and was not associated with stroke.

- The odds of heart disease were $45 \%$ higher in persons with $\mathrm{OA}$, compared with age-matched persons without $O A$ and most associations appeared stronger in women than in men.

Strengths and limitations of this study

- We used a large and representative sample from the Canadian population.

- The results were adjusted for age, body mass index, income, education, physical activity, smoking status, fruit and vegetable consumption, pain medication use, diabetes, hypertension and chronic obstructive pulmonary disease.

- The cross-sectional data prevented us from assessing the temporal exposure-outcome sequence between OA and CVD.

- Self-reported data tend to contain both falsepositive and false-negative values, and therefore may introduce bias in the estimates.

\section{INTRODUCTION}

Osteoarthritis (OA) is a highly prevalent chronic disorder and a leading cause of disability among the elderly. ${ }^{1-3}$ Although the 
prevalence estimates for OA show wide variability depending on the age and sex of the studied population, the case identification used, and the joint sites included, in British Columbia (BC), Canada, overall prevalence of OA was 10.8 per cent, ${ }^{4}$ and in the USA about $10-12 \%$ of the population have $\mathrm{OA}^{5-7} \mathrm{OA}$ is associated with increasing age, female sex, obesity, injury in the joints, occupation and physical activity. ${ }^{14-8}$ Coronary heart diseases (CHDs) including myocardial infarction (MI) and angina and other types of cardiovascular diseases (CVDs) such as congestive heart failure (CHF) and stroke are the leading causes of morbidity and mortality in the general population. ${ }^{9}{ }^{10}$ Well-known factors that are associated with CVD include age, sex, obesity, hypertension, cholesterol, income, ethnicity, exercise, smoking and diets. ${ }^{9-12}$ Studies have shown that systemic inflammation can increase the risk of CVD. ${ }^{13}{ }^{14}$ Rheumatic diseases other than OA are characterised by acute or chronic inflammation and they have been linked to an increased risk of CVD. ${ }^{15}$ Muscle weakness was also observed as a risk factor and comorbidity among individuals with CVD. ${ }^{16}$ Another risk factor is physical inactivity. There is substantial evidence to indicate that physically active individuals have lower rates of CVD. ${ }^{17} 18$

Although OA is the most common rheumatic disease, very little is known about the relationship between OA and CVD. OA may be associated with CVD for a number of reasons. The first is physical inactivity. With OA progression, severe pain in the joints makes patients less physically active compared to individuals without arthritis. ${ }^{19}$ Recent studies have shown that immobility resulting from arthritis may increase CVD risk among elderly patients and thus shorten the lifespan. ${ }^{20}$ The second is chronic inflammation. Although OA is often referred to as a degenerative disease, recent studies have shown that synovial inflammation plays a role in the development of early stages of $\mathrm{OA}^{21}$ The third is muscle weakness. Muscle weakness is more common among individuals with OA than healthy controls ${ }^{22} 23$ and muscle weakness has been observed as a risk factor and comorbid condition among individuals with CVD. ${ }^{16}$ Furthermore, nonsteroidal anti-inflammatory drugs (NSAIDs), commonly used to treat OA-related pain, are associated with increased risk of CVD. ${ }^{24}{ }^{25}$ To sum up, the reasons why OA may be associated with CVD include physical inactivity, chronic inflammation, muscle weakness and NSAID use among individuals with OA.

The relationship between OA and CVD has not been studied extensively in population-based studies. However, there is some evidence that OA patients have higher rates of CVD and its risk factors than individuals without OA. Using the US National Health and Nutrition Examination Survey data, Singh $e t a l^{26}$ found that adults with OA had a high prevalence of cardiovascular risk factors. Recently, Ong et $a l^{27}$ observed higher odds of CVD among OA cases compared with non-arthritis controls. In a case-control study of general practice consulters in England and Wales, Kadam et $a t^{28}$ observed increased ORs of ischaemic heart disease (IHD), angina and heart failure among OA cases relative to non-OA controls. There is also some evidence that OA patients are at an increased risk of cardiovascular mortality. In a cohort study in England, patients with OA had increased mortality compared with the general population due to CVD, diabetes, dementia and cancer. ${ }^{29}$ After reviewing the literature on mortality and OA, Hochberg ${ }^{30}$ found higher mortality among OA patients compared with the general population.

The large sample size of the Canadian Community Health Survey (CCHS) provides an ideal opportunity to examine the possible association between $\mathrm{OA}$ and heart disease and other cardiovascular outcomes. The main objective of this study was to examine the association between OA and CVD using CCHS data. We examined the association of OA with self-reported heart disease, $\mathrm{MI}$, angina, $\mathrm{CHF}$ and stroke. We hypothesised that $\mathrm{OA}$ is strongly associated with CVD and the association is due, at least partially, to immobility, chronic inflammation, muscle weakness, NSAID use and other lifestyle changes due to OA. Given that $\mathrm{OA}$ is a common condition among the elderly, a better understanding of the relationship between OA and CVD could help further investigation of potential biological and behavioural mechanisms underlying this association and also inform future OA management strategies.

\section{METHODOLOGY}

\section{Data source and study population}

This study used data from the CCHS cycles 1.1, 2.1 and 3.1 conducted in years 2000/2001, 2002/2003 and 2004/2005, respectively. Performed by Statistics Canada, CCHS contains nationally representative data on health determinants, health status and health system utilisation. This cross-sectional survey used a multistage stratified cluster probability sampling in which a dwelling was the final sampling unit. The survey sample was stratified by province/territory and urban versus rural regions within each province/territory. Three sampling frames were used to select the sample of households: $48-49 \%$ of the sample came from an area frame, $50 \%$ came from a list frame of telephone numbers and 1-2\% came from a Random Digit Dialling sampling frame. Persons in the households selected from the telephone list frame were interviewed from centralised call centres. Those selected from the area frame were interviewed face-to-face by decentralised field interviewers. In some situations, field interviewers completed some interviews or part of an interview by telephone. Sampling was designed to represent $98 \%$ of the Canadian population aged 12 years or more who lived in private dwellings in the 10 provinces and the three territories. A detailed description of the survey design, sample frame and interviewing procedures may be found elsewhere. ${ }^{31}$ In all three CCHS cycles, Statistics Canada included the type of arthritis as 
a separate question in all jurisdictions. Owing to very low prevalence of OA and CVD among individuals aged 12-19 years, we decided to exclude them from the study. The three cycles of CCHS used different and nonoverlapping samples. There were 113323 respondents in the 2000/2001 survey, 115548 respondents in the 2002/2003 survey and 115915 respondents in the 2004/ 2005 survey, respectively, aged 20 years and older.

\section{Concepts and measures}

\section{$O A$ and CVD}

The main independent variable in this study was OA and the primary outcome was heart disease. Specific cardiovascular conditions such as MI, angina, CHF or stroke were secondary outcomes in this study. In CCHS data, OA was assessed by asking two questions. The first question was "Now I'd like to ask about certain chronic health conditions that have lasted 6 months or more and have been diagnosed by a health professional. Do you have arthritis or rheumatism?" The second question was "What type of arthritis?" and the four response options were Rheumatoid arthritis, OA, other, and do not know". Since the second question is restricted to only arthritis patients, over-reporting of $\mathrm{OA}$ is minimised. In the sample, we observed that out of the total arthritis cases, $81 \%$ reported that they had OA. The prevalence of heart disease was assessed by the following question "Now I'd like to ask about certain chronic health conditions that have lasted 6 months or more and have been diagnosed by a health professional. Do you have heart disease?" In CCHS cycles 1.1 and 2.1, persons reporting heart disease were asked specific questions about MI, angina, CHF and stroke: "Have you ever had a heart attack (damage to the heart muscle)?", "Do you currently have angina (chest pain, chest tightness)?", and "Do you currently have CHF (inadequate heartbeat, fluid build-up in the lungs or legs)?". In addition, CCHS includes the question of "Do you suffer from the effects of a stroke?" These specific questions provide the data to perform disease-specific analyses using each of these four health conditions as outcomes using CCHS cycles 1.1 and 2.1 .

\section{Covariates and confounders}

We have included demographic, socioeconomic and health behaviour variables to serve as control variables in regression modelling. Selection of these variables was based on previous research. ${ }^{12}$ The sociodemographic variables were age, sex, body mass index (BMI), education and household income. Health behaviours included physical activity, smoking status, fruits and vegetable consumption and pain medication use. Chronic health conditions such as chronic obstructive pulmonary disease (COPD), diabetes and hypertension were included as covariates. Cigarette smoking was a derived variable which was measured using 18 different questions regarding the frequency and length of time of smoking. We categorised the smoking variable as current smoker, former smoker and non-smoker. BMI was expressed in $\mathrm{kg} / \mathrm{m}^{2}$ and was calculated from self-reported heights and weights. The classification was made based on the four following BMI subgroups: underweight $(\mathrm{BMI}<18.5)$, normal $(18.5 \leq \mathrm{BMI}<24.9)$, overweight $(25.0 \leq \mathrm{BMI}<29.9)$ and obese $(B M I \geq 30.0)$. Physical activity was derived from the self-reported level of activity and the amount of time spent on physical activities for 3 months prior to the survey. These responses were categorised as being active, moderately active or inactive. The level of education with four categories, such as less than secondary school, secondary school graduate, some postsecondary education, postsecondary graduate and household income level, were considered as socioeconomic status (SES) characteristics. Daily consumption of fruits and vegetables was divided into three categories such as less than 3 servings/day, 4-6 servings/day and more than 6 servings per day. Pain medication use was assessed by asking two questions: (1) "In the past month, did you take pain relievers such as aspirin or Tylenol (including arthritis medicine and anti-inflammatories)?" and (2) "In the past month, did you take codeine, Demerol or morphine?" Among all participants, BMI was missing for $13 \%$, education status was missing for $2.5 \%$, income was missing for $21 \%$, and fruit and vegetable consumption was missing for $17.5 \%$ of the respondents. We imputed missing BMI values using linear regression on age and sex for complete cases, and the missing values for other categorical variables were imputed by taking the mode values after adjusting for age and sex. ${ }^{32}$

\section{Statistical analysis}

In this cross-sectional study, to control for age and sex in the $\mathrm{OA}$ and non-OA individuals in the sample, we selected one non-OA individual for each OA patient by matching age category, sex and CCHS cycles.

Initially, we attempted to select three non-OA individuals for each OA case by matching for age, sex and CCHS cycles. However, we observed a high prevalence of OA in the older age groups (ie, more than $40 \%$ among individuals aged $>70$ have $\mathrm{OA}$ ), and therefore it was not possible to match exactly three non-OA individuals in the older age groups. With the 1:3 approximately matched samples, we repeated the entire analyses as part of a sensitivity analysis. Frequency and percentages were calculated for all variables prior to modelling the associations between OA and CVD. Unadjusted effects of OA on CVD were calculated using logistic regression where age and sex were included in the models. Multivariable logistic regression models were used to assess the association between OA and heart disease, MI, angina, CHF and stroke after controlling for the potential confounding variables. All statistical analyses were performed using SAS V.9.3 (SAS Institute, Cary, North Carolina, USA). Statistics Canada produced sampling weights for each of the study participants of CCHS. All estimates were weighted to approximate the distribution of demographic variables in the overall Canadian 
population. Since CCHS uses a complex sampling design, the CIs of the estimates were adjusted using a design effect of $2 .^{31} 33$

\section{RESULTS}

The sample characteristics of the OA and non-OA respondents who were included in the analyses are presented in table 1. The demographic characteristics, social status and health conditions were compared between 40817 OA cases and matched non-OA respondents. All these comparisons were statistically significant with $\mathrm{p}<0.01$. The mean age of $\mathrm{OA}$ cases was 66 years and $71.6 \%$ were women. In the 1:1 matched samples, we observed that OA patients were significantly more overweight and obese, had more diabetes, hypertension and were more diagnosed with COPD, compared to non-OA respondents. The percentage of participants who reported being physically active was $16.9 \%$ in $\mathrm{OA}$ vs $18.6 \%$ in non-OA and the percentage moderately active was similar in both groups. OA cases used more pain medications than non-OA individuals.

Table 1 Sample characteristics (per cent) of osteoarthritis (OA) and non-OA individuals by exposure status

\begin{tabular}{|c|c|c|c|}
\hline Characteristics & OA cases $(n=40817)$ & $1: 1$ matched non-OA $(n=40817)$ & $1: 3$ matched non-OA $(n=109450)$ \\
\hline \multicolumn{4}{|l|}{ Age } \\
\hline 20-39 & 4.6 & 4.5 & 5.1 \\
\hline $40-49$ & 9.1 & 9.0 & 10.1 \\
\hline $50-59$ & 19.4 & 19.6 & 21.8 \\
\hline $60-69$ & 25.4 & 25.3 & 26.3 \\
\hline $70-79$ & 24.7 & 25.0 & 22.0 \\
\hline$\geq 80$ & 16.9 & 16.6 & 14.8 \\
\hline Women & 71.6 & 71.6 & 68.2 \\
\hline \multicolumn{4}{|l|}{ Body mass index } \\
\hline$<18.4$ & 1.7 & 1.9 & 1.8 \\
\hline $18.5-24.9$ & 30.7 & 35.9 & 36.4 \\
\hline 25-29.9 & 30.5 & 29.7 & 30.8 \\
\hline$\geq 30$ & 20.5 & 14.3 & 14.6 \\
\hline Missing & 16.6 & 18.2 & 16.4 \\
\hline COPD & 3.0 & 2.0 & 2.0 \\
\hline Diabetes & 11.5 & 9.9 & 9.4 \\
\hline Hypertension & 37.6 & 32.1 & 30.4 \\
\hline \multicolumn{4}{|l|}{ Physical activity } \\
\hline Active & 16.9 & 18.6 & 19.0 \\
\hline Moderately active & 22.4 & 22.9 & 23.3 \\
\hline Inactive & 60.6 & 58.5 & 57.7 \\
\hline Pain medication use & 12.9 & 10.5 & 10.5 \\
\hline \multicolumn{4}{|l|}{ Smoking } \\
\hline Currently smoke & 19.7 & 19.0 & 19.8 \\
\hline Former smoker & 49.6 & 46.6 & 47.2 \\
\hline Non-smoker & 30.7 & 34.4 & 33.0 \\
\hline \multicolumn{4}{|l|}{ Fruits and vegetables } \\
\hline 0-3 serving daily & 16.1 & 15.6 & 15.3 \\
\hline 4-6 serving daily & 43.1 & 43.7 & 43.6 \\
\hline $6+$ serving daily & 23.7 & 22.3 & 22.6 \\
\hline Not stated & 17.1 & 18.5 & 18.5 \\
\hline \multicolumn{4}{|l|}{ Income } \\
\hline$<29999$ & 32.4 & 29.1 & 27.4 \\
\hline 30000-49999 & 21.7 & 20.6 & 20.6 \\
\hline 50000-79999 & 17.1 & 16.9 & 17.7 \\
\hline$\geq 80000$ & 11.8 & 13.3 & 14.4 \\
\hline Not stated & 17.0 & 20.2 & 19.9 \\
\hline \multicolumn{4}{|l|}{ Education } \\
\hline Less than secondary & 35.4 & 36.4 & 34.6 \\
\hline Secondary graduate & 15.4 & 15.9 & 16.2 \\
\hline Some postsecondary & 6.1 & 5.3 & 5.5 \\
\hline Postsecondary graduation & 41.4 & 40.0 & 41.3 \\
\hline Not stated & 1.8 & 2.4 & 2.4 \\
\hline
\end{tabular}


The proportion of non-smokers was lower in the OA group. Percentages in the income, education and fruit and vegetable categories were slightly but significantly different among OA and non-OA respondents.

Both the unadjusted and adjusted effects of OA on the prevalence of heart disease for men, women and total are presented in table 2. In the multivariable model, those who had OA exhibited increased odds of prevalent heart disease (OR 1.45, 95\% CI 1.36 to 1.54 ) compared to non-OA individuals after controlling for age, sex, income, education, BMI, physical activity, smoking, fruit and vegetable consumption, pain medication use, COPD, hypertension and diabetes. We observed a borderline significant interaction between sex and OA ( $p$ value $=0.058$ ) in the multivariable model, and therefore we decided to perform separate analyses for men and women. ORs $(95 \% \mathrm{CI})$ for heart disease were 1.35 (1.21 to 1.50 ) and 1.50 (1.39 to 1.64 ) among men and women with OA, respectively. Income, education, BMI, physical activity, smoking, medication use,
COPD, hypertension and diabetes were associated with heart disease as expected.

Multivariable logistic regression models were fitted to estimate the effects of OA on the prevalence of MI, angina, CHF and stroke for the total sample, and separately for men and women, and are presented in table 3. OA was significantly associated with $\mathrm{MI}$, angina and CHF (OR (95\% CI), 1.28 (1.15 to 1.44 ), 1.83 (1.62 to 2.06) and 1.72 (1.46 to 2.01 ), respectively) compared with subjects without OA. In sex-specific analyses, OR was highest for angina (OR (95\% CI), 1.76 (1.43 to 2.17) and 1.85 (1.59 to 2.14) for men and women, respectively), followed by CHF. OA showed significantly increased OR for MI among women only but did not show a significant association with stroke. In the 1:3 matched OA versus non-OA samples, we observed similar ORs, but with narrower CIs. OR (95\% CI) of overall heart disease was 1.44 (1.36 to 1.51) among men, and 1.46 (1.37 to 1.56 ) among women in 1:3 matched samples (data not shown).

Table 2 ORs and 95\% Cls of heart diseases for osteoarthritis and non-osteoarthritis 1:1 matched samples by age and sex

\begin{tabular}{|c|c|c|c|c|}
\hline Variables & Levels & Overall OR $(95 \% \mathrm{Cl})$ & Men OR $(95 \% \mathrm{Cl})$ & Women OR $(95 \% \mathrm{Cl})$ \\
\hline Osteoarthritis unadjusted & Yes & $1.54(1.45$ to 1.64$)$ & 1.47 (1.33 to 1.63$)$ & 1.59 (1.47 to 1.72$)$ \\
\hline Osteoarthritis adjusted & Yes & $1.45(1.36$ to 1.54$)$ & 1.35 (1.21 to 1.50$)$ & 1.51 (1.39 to 1.64$)$ \\
\hline \multirow[t]{6}{*}{ Age } & $20-39$ & Reference & Reference & Reference \\
\hline & $40-49$ & 2.24 (1.50 to 3.33$)$ & 1.67 (0.93 to 3.02$)$ & 2.62 (1.52 to 4.52$)$ \\
\hline & $50-59$ & 4.28 (2.95 to 6.21$)$ & 5.58 (3.27 to 9.52$)$ & 3.41 (2.03 to 5.72$)$ \\
\hline & $60-69$ & 7.19 (4.97 to 10.41$)$ & 8.47 (4.98 to 14.41$)$ & 6.09 (3.64 to 10.19$)$ \\
\hline & $70-79$ & 11.87 (8.19 to 17.20$)$ & 13.29 (7.79 to 22.69$)$ & $10.28(6.14$ to 17.21$)$ \\
\hline & $\geq 80$ & $19.33(13.30$ to 28.11$)$ & 18.10 (10.51 to 31.18$)$ & 18.35 (10.92 to 30.82$)$ \\
\hline \multirow[t]{4}{*}{ Income } & $<30000$ & Reference & Reference & Reference \\
\hline & $30000-50000$ & $0.93(0.85$ to 1.01$)$ & $0.90(0.78$ to 1.04$)$ & $0.94(0.85$ to 1.04$)$ \\
\hline & $50000-80000$ & 0.82 (0.74 to 0.90$)$ & 0.89 (0.76 to 1.04$)$ & 0.77 (0.68 to 0.87$)$ \\
\hline & $\geq 80000$ & 0.69 (0.62 to 0.78$)$ & 0.65 (0.55 to 0.78$)$ & 0.69 (0.59 to 0.81$)$ \\
\hline \multirow[t]{4}{*}{ Education } & Elementary & Reference & Reference & Reference \\
\hline & Secondary & $0.87(0.79$ to 0.96$)$ & $0.97(0.81$ to 1.15$)$ & $0.84(0.74$ to 0.94$)$ \\
\hline & Some postsecondary & 0.91 (0.78 to 1.06$)$ & 0.98 (0.77 to 1.26$)$ & 0.88 (0.73 to 1.07$)$ \\
\hline & Graduation & 0.96 (0.89 to 1.03$)$ & $1.05(0.92$ to 1.19$)$ & $0.92(0.83$ to 1.01$)$ \\
\hline \multirow[t]{4}{*}{ Body mass index } & $<18.4$ & 1.05 (0.82 to 1.35$)$ & 0.85 (0.45 to 1.58$)$ & 1.06 (0.81 to 1.39$)$ \\
\hline & $18.5-24.9$ & Reference & Reference & Reference \\
\hline & $25-29.9$ & $0.99(0.92$ to 1.08$)$ & 1.09 (0.95 to 1.24$)$ & 0.94 (0.84 to 1.04$)$ \\
\hline & $\geq 30$ & 1.14 (1.03 to 1.26$)$ & $1.23(1.04$ to 1.45$)$ & 1.09 (0.96 to 1.23$)$ \\
\hline \multirow[t]{3}{*}{ Physical activity } & Active & Reference & Reference & Reference \\
\hline & Moderate & $1.11(0.99$ to 1.24$)$ & 1.19 (1.01 to 1.41$)$ & 1.07 (0.91 to 1.25$)$ \\
\hline & Inactive & 1.33 (1.21 to 1.47$)$ & $1.28(1.11$ to 1.48$)$ & $1.37(1.20$ to 1.57$)$ \\
\hline \multirow[t]{3}{*}{ Smoking } & Non-smoker & Reference & Reference & Reference \\
\hline & Currently & $1.16(1.04$ to 1.29$)$ & 1.40 (1.16 to 1.69$)$ & 1.09 (0.96 to 1.25$)$ \\
\hline & Former & 1.19 (1.11 to 1.29$)$ & 1.39 (1.20 to 1.61$)$ & 1.16 (1.06 to 1.26$)$ \\
\hline \multirow{3}{*}{ Fruits and vegetables } & 0-3 Servings daily & Reference & Reference & Reference \\
\hline & 4-6 Servings daily & $1.03(0.96$ to 1.10$)$ & 1.10 (0.99 to 1.23$)$ & 0.98 (0.89 to 1.07$)$ \\
\hline & $6+$ Servings daily & $1.15(1.07$ to 1.25$)$ & $1.48(1.31$ to 1.68$)$ & $1.01(0.91$ to 1.11$)$ \\
\hline Pain medication use & Yes & $1.13(1.03$ to 1.24$)$ & 1.22 (1.04 to 1.43$)$ & 1.08 (0.96 to 1.21$)$ \\
\hline Hypertension & Yes & 1.98 (1.86 to 2.12$)$ & $1.92(1.72$ to 2.14$)$ & 2.01 (1.84 to 2.18$)$ \\
\hline COPD & Yes & 2.79 (2.39 to 3.26$)$ & 2.98 (2.35 to 3.78 ) & 2.70 (2.19 to 3.31$)$ \\
\hline Diabetes & Yes & $1.90(1.75$ to 2.07$)$ & 1.80 (1.57 to 2.06$)$ & $1.96(1.76$ to 2.19$)$ \\
\hline
\end{tabular}


Table 3 Adjusted and unadjusted OR and $95 \% \mathrm{Cl}$ of specific cardiovascular outcomes for osteoarthritis (OA) in the age-matched and sex-matched sample

\begin{tabular}{lllll}
\hline Outcome & Model & Overall OR (95\% Cl) & Men OR (95\% Cl) & Women OR (95\% CI) \\
\hline Myocardial infarction $(n=3197)$ & OA adjusted & $1.28(1.15$ to 1.44$)$ & $1.08(0.91$ to 1.28$)$ & $1.49(1.28$ to 1.75$)$ \\
& OA unadjusted & $1.38(1.23$ to 1.54$)$ & $1.19(1.01$ to 1.40$)$ & $1.56(1.34$ to 1.82$)$ \\
Angina $(n=3143)$ & OA adjusted & $1.83(1.62$ to 2.06$)$ & $1.76(1.43$ to 2.17$)$ & $1.85(1.59$ to 2.14$)$ \\
& OA unadjusted & $1.94(1.73$ to 2.18$)$ & $1.94(1.58$ to 2.38$)$ & $1.94(1.68$ to 2.24$)$ \\
Congestive heart failure $(n=1586)$ & OA adjusted & $1.72(1.46$ to 2.01$)$ & $1.50(1.13$ to 1.97$)$ & $1.81(1.49$ to 2.21$)$ \\
& OA unadjusted & $1.84(1.57$ to 2.15$)$ & $1.71(1.31$ to 2.23$)$ & $1.91(1.57$ to 2.31$)$ \\
Stroke $(n=1112)$ & OA adjusted & $1.11(0.95$ to 1.29$)$ & $1.08(0.83$ to 1.40$)$ & $1.13(0.93$ to 1.37$)$ \\
& OA unadjusted & $1.20(1.03$ to 1.39$)$ & $1.14(0.89$ to 1.48$)$ & $1.23(1.01$ to 1.48$)$ \\
\hline
\end{tabular}

Adjusted ORs were obtained after controlling for age, sex, income, education, body mass index, physical activity, smoking, fruit and vegetable consumptions, pain medication use, chronic obstructive pulmonary disease, hypertension and diabetes.

\section{DISCUSSION}

This study sought to describe the association between $\mathrm{OA}$ and the prevalence of heart disease, MI, angina, $\mathrm{CHF}$ and stroke using CCHS data. In this populationbased study, adjusted for age, BMI, income, education, physical activity, smoking status, fruit and vegetable consumption, medication use, diabetes, hypertension and COPD, we found statistically significant positive associations of OA with heart disease, angina and CHF among both men and women. These associations were higher among women than men. OA was positively associated with MI among women only and there was no significant association between OA and stroke.

There is some evidence that OA patients have higher rates of CVD and its risk factors. Singh et $a l^{26}$ found that adults with OA have a high prevalence of cardiovascular risk factors. Kadam et $a t^{8}$ observed $73 \%, 36 \%$ and $28 \%$ higher odds for IHD, angina and heart failure, respectively, among OA cases compared to non-OA controls in a case-control study of consultations in general practices in England and Wales. Recently, Ong et $a l^{27}$ observed significantly higher odds for self-reported CVD, CHD and angina among OA cases compared to their non-OA counterparts in a cross-sectional study using US National Health and Nutrition Examination Survey data. Our cross-sectional study results of statistically significant associations between $\mathrm{OA}$ and prevalent heart disease, MI, angina and $\mathrm{CHF}$ are consistent with the previous findings. Similar to our data, stroke was not associated with OA in Ong et $a l \mathrm{~s}^{27}$ study. This study included confounding and mediating variables such as physical activity, fruit and vegetable consumption and pain medication use, which were not adjusted for in the previous studies. In addition, our present study is generalisable to Canada because the surveys were conducted in a representative sample of the Canadian population. Other published data have shown that OA patients had increased mortality due to CVD. ${ }^{29}{ }^{30}$ Nuesch $e t a l^{29}$ performed a population-based cohort study to examine all-cause and disease-specific mortality in patients with OA of the hip and knee, and concluded that OA patients had excess all-cause mortality compared with the general population and $71 \%$ excess standard mortality ratio of CVD. Hochberg ${ }^{30}$ reviewed the literature on mortality in OA and found several studies, suggesting an increased risk of death. ${ }^{34-37}$ These studies had small sample sizes and other limitations. One study recruited patients from hospitals or medical practices; another study investigated patients after total knee replacement, but not all relevant factors were adjusted for in the multivariable analyses. We observed a significant positive association between $\mathrm{OA}$ and the prevalence of heart disease after controlling for several covariates that were not included in previous studies of OA and mortality.

Among the covariates, BMI, income, education, physical activity, smoking status, medication use, diabetes, hypertension and COPD were associated with heart disease, MI, angina and $\mathrm{CHF}$ in both men and women as expected. We observed that higher income and higher education groups had lower ORs, providing support for previous studies showing that people with low SES are at high risk of CVD. ${ }^{38}$ BMI was statistically significant in the multivariable models for all four health outcomes in our study, which shows agreement with previous results. ${ }^{39} 40$ The physically inactive and moderately active groups had significantly higher ORs than the active group in the multivariable models of heart disease and for all specific outcomes, which supports the findings of previous studies that show physical activity to be inversely associated with heart disease prevalence, controlling for covariates and confounders. ${ }^{17} 18$ Smoking is a well-known risk factor for heart disease and we observed a significantly higher risk in current and former smokers in the multivariable models.

The cross-sectional nature of the CCHS data prevented us from assessing the temporal exposure -outcome sequence between OA and heart disease. Until the relationship between $\mathrm{OA}$ and CVD is measured prospectively in longitudinal studies any association suggested by cross-sectional analysis remain hypothesisgenerating. Self-reported data tend to contain both falsepositive and false-negative values and therefore may result in bias in the estimates. Obesity was determined from self-reported height and weight. Comparisons of 
measured and self-reported BMI have suggested that self-reported values may underestimate the true prevalence of obesity by $9 \%$ for men and $6 \%$ for women. ${ }^{41}$ However, this small underreporting alone would not change the $\mathrm{OA}$ and heart disease relationships. Missing values for BMI, education, income and fruit and vegetable consumption were imputed. Although the imputed values may not reflect the true distribution of these variables in the sample, the effect on the results is probably small given the relatively small proportion of missing values in our data.

The observed small differences between the unadjusted and adjusted ORs suggest that the confounding variables had minimal effects on the relationship between OA and CVD. In the present analysis, we were unable to study the possible mediating role of muscle weakness and chronic inflammation. Examining the effect of OA after adjusting for these factors in future cohort studies might help elucidate the causal mechanisms for the observed associations. Other potential intermediate variables, such as physical activity and pain medication use, were included in this study. However, the differences in the distribution of these variables among $\mathrm{OA}$ and non-OA respondents in our data were small. As a result, there was no notable difference in the ORs with and without these variables included in the models (data not shown). Although these data do not support the mediating role of physical activity and pain medication use in the association between OA and CVD, it is important to note that our analysis was limited by the cross-sectional nature of the data and possible errors in reporting.

The exploration of the relationship of $\mathrm{OA}$ with heart disease, MI, CHF and angina remains a promising and important area of research. Since OA is a very common health condition, an association between OA and CVD would be important from a public health perspective. This study identified possible causal associations and raised further questions for subsequent research. However, the results are preliminary, and therefore conclusions should be clearly conditional on confirmation in future studies. More prospective studies are needed to understand the temporal ordering of the relationship between exposure to OA and the incidence of CVD.

In conclusion, our study findings suggest that OA may be associated with an increased risk of heart disease in a broadly representative population-based context. Our data demonstrate positive associations between $\mathrm{OA}$ and angina, CHF and MI. Although these conclusions are conditional on further confirmation, there is an increased potential for more CVD among adults with OA. This study provides a rationale for further investigation of the association between $\mathrm{OA}$ and heart disease in longitudinal studies for possible biological and behavioural mechanisms that may be responsible for this association.

\section{Author affiliations}

${ }^{1}$ School of Population and Public Health, University of British Columbia Vancouver, British Columbia, Canada
${ }^{2}$ Arthritis Research Centre of Canada, Richmond, British Columbia, Vancouver, Canada

${ }^{3}$ Department of Medicine, University of British Columbia, Vancouver, British Columbia, Canada

${ }^{4}$ Department of Health Sciences, Simon Fraser University, Burnaby, British Columbia, Canada

${ }^{5}$ Centre for Health Evaluation and Outcome Sciences, Vancouver, British Columbia, Canada

Contributors MMR, JAK, AHA, JC and CHG contributed to the concept and design of the study. MMR and JAK performed the data acquisition and statistical analyses. MMR conducted the literature search and wrote the manuscript. JAK contributed to the initial revision of the manuscript. JAK, $\mathrm{AHA}, \mathrm{JC}$ and $\mathrm{CHG}$ contributed to the critical revision of the manuscript before publication. MMR is the guarantor.

Funding This research received no specific grant from any funding agency in the public, commercial or not-for-profit sectors.

Competing interests MMR received a graduate training award from the Canadian Arthritis Network/The Arthritis Society and a doctoral training award from the Canadian Institutes of Health Research.

Data sharing statement No additional data are available.

STROBE statement This observational study was performed and the manuscript was written according to the STROBE guidelines.

\section{REFERENCES}

1. Felson DT, Zhang Y. An update on the epidemiology of knee and hip osteoarthritis with a view to prevention. Arthritis Rheum 1998;41:1343-55.

2. Lawrence RC, Helmick CG, Arnett FC, et al. Estimates of the prevalence of arthritis and selected musculoskeletal disorders in the United States. Arthritis Rheum 1998;41:778-99.

3. Lawrence RC, Felson DT, Helmick CG, et al. Estimates of the prevalence of arthritis and other rheumatic conditions in the United States. Part II. Arthritis Rheum 2008;58:26-35.

4. Kopec JA, Rahman MM, Berthelot JM, et al. Descriptive epidemiology of osteoarthritis in British Columbia, Canada. J Rheumatol 2007;34:386-93.

5. Hunter DJ. Osteoarthritis. Best Pract Res Clin Rheumatol 2011;25:801-14.

6. Dunlop DD, Manheim LM, Song J, et al. Arthritis prevalence and activity limitations in older adults. Arthritis Rheum 2001;44:212-21.

7. Suri P, Morgenroth DC, Hunter DJ. Epidemiology of osteoarthritis and associated comorbidities. PMR 2012;4:S10-19.

8. Felson DT, Lawrence RC, Hochberg MC, et al. Osteoarthritis: new insights. Part 2: treatment approaches. Ann Intern Med 2000;7:726-37.

9. Yusuf S, Reddy S, Ounpuu S, et al. Global burden of cardiovascular diseases: part I: general considerations, the epidemiologic transition, risk factors, and impact of urbanization. Circulation 2001;104:2746-53.

10. Yusuf S, Reddy S, Ounpuu S, et al. Global burden of cardiovascular diseases: part II: variations in cardiovascular disease by specific ethnic groups and geographic regions and prevention strategies. Circulation 2001;104:2855-64.

11. Tanuseputro P, Manuel DG, Leung M, et al. Risk factors for cardiovascular disease in Canada. Can J Cardiol 2003;19:1249-59.

12. Lee DS, Chiu M, Manuel DG, et al. Trends in risk factors for cardiovascular disease in Canada: temporal, socio-demographic and geographic factors. CMAJ 2009;181:E55-66.

13. van Leuven SI, Franssen R, Kastelein JJ, et al. Systemic inflammation as a risk factor for atherothrombosis. Rheumatology (Oxford) 2008;47:3-7.

14. Hansson GK, Hermansson A. The immune system in atherosclerosis. Nat Immunol 2011;12:204-12.

15. Shoenfeld $Y$, Gerli R, Doria A, et al. Accelerated atherosclerosis in autoimmune rheumatic diseases. Circulation 2005;112:3337-47.

16. Walston J, Mcburnie MA, Newman A, et al. Frailty and activation of the inflammation and coagulation systems with and without clinical comorbidities: results from the Cardiovascular Health Study. Arch Intern Med 2002;162:3-11.

17. Shiroma EJ, Lee IM. Physical activity and cardiovascular health: lessons learned from epidemiological studies across age, gender, and race/ethnicity. Circulation 2010;122:743-52. 
18. Li J, Siegrist J. Physical activity and risk of cardiovascular diseasea meta-analysis of prospective cohort studies. Int J Environ Res Public Health 2012:9:391-407.

19. Hootman JM, Macera CA, Ham SA, et al. Physical activity levels among the general US adult population and in adults with and without arthritis. Arthritis Rheum 2003;49:129-35.

20. Landi F, Liperoti R, Russo A, et al. Disability, more than multimorbidity, was predictive of mortality among older persons aged 80 years and older. J Clin Epidemiol 2010;63:752-9.

21. Sellam J, Berenbaum $F$. The role of synovitis in pathophysiology and clinical symptoms of osteoarthritis. Nat Rev Rheumatol 2010;6:625-35.

22. Palmieri-Smith RM, Thomas AC, Karvonen-Gutierrez C, et al. Isometric quadriceps strength in women with mild, moderate, and severe knee osteoarthritis. Am J Phys Med Rehabil 2010;89:541-8.

23. Slemenda C, Heilman DK, Brandt KD, et al. Reduced quadriceps strength relative to body weight: a risk factor for knee osteoarthritis in women. Arthritis Rheum 1998:41:1951-9.

24. Mcgettigan P, Henry D. Cardiovascular Risk and Inhibition of Cyclooxygenase. JAMA 2006;296:1633-44.

25. Trelle S, Reichenbach S, Wandel S, et al. Cardiovascular safety of non-steroidal anti-inflammatory drugs: network meta-analysis. BMJ 2011;342:c7086.

26. Singh G, Miller JD, Lee FH, et al. Prevalence of cardiovascular disease risk factors among US adults with selfreported osteoarthritis: data from the third National Health and Nutrition Examination Survey. Am J Manag Care 2002;8:S383-91.

27. Ong KL, Wu BJ, Cheung BM, et al. Arthritis: its prevalence, risk factors, and association with cardiovascular diseases in the United States, 1999 to 2008. Ann Epidemiol 2013;23:80-6.

28. Kadam UT, Jordan K, Croft PR. Clinical comorbidity in patients with osteoarthritis: a case-control study of general practice consulters in England and Wales. Ann Rheum Dis 2004;63:408-14.

29. Nüesch E, Dieppe $P$, Reichenbach $S$, et al. All cause and disease specific mortality in patients with knee or hip osteoarthritis: population based cohort study. BMJ 2011;342:d1165.
30. Hochberg MC. Mortality in osteoarthritis. Clin Exp Rheumatol 2008;26:S120-4.

31. Béland Y. Canadian Community Health Survey -methodological overview. Health Reports 2002;13:9-14.

32. Little RJA, Rubin DB. Statistical analysis with missing data. New York: J. Wiley \& Sons, 1987.

33. Rust KF, Rao JNK. Variance estimation for complex surveys using replication techniques. Stat Methods Med Res 1996;5:283-310.

34. Cerhan JR, Wallace RB, el-Khoury GY, et al. Decreased survival with increasing prevalence of full-body, radiographically defined osteoarthritis in women. Am J Epidemiol 1995;141:225-34.

35. Haara MM, Manninen $\mathrm{P}$, Kröger $\mathrm{H}$, et al. Osteoarthritis of finger joints in finns aged 30 or over: prevalence, determinants, and association with mortality. Ann Rheum Dis 2003;62:151-8.

36. Watson DJ, Rhodes T, Guess HA. All-cause mortality and vascular events among patients with rheumatoid arthritis, osteoarthritis, or no arthritis in the UK General Practice Research Database. $J$ Rheumatol 2003;30:1196-202.

37. Monson RR, Hall AP. Mortality among arthritis. J Chron Dis 1976;29:459-67.

38. Albert MA, Glynn RJ, Buring J. Impact of traditional and novel risk factors on the relationship between socioeconomic status and incident cardiovascular events. Circulation 2006;114:2619-26.

39. Wolk R, Berger P, Lennon RJ, et al. Body mass index: a risk factor for unstable angina and myocardial infarction in patients with angiographically confirmed coronary artery disease. Circulation 2003;108:2206-11.

40. Eckel RH. Obesity and heart disease: a statement for healthcare professionals from the nutrition committee, American Heart Association. Circulation 1997;96:3248-50.

41. Shields M, Gorber SC, Tremblay MS. Estimates of obesity based on self-report versus direct measures. Health Rep 2008;19:61-76. 